Emotional Distress, Quality Of Life, Self-compassion, Mindfulness, And Optimism In Patients With Hematologic Cancer Waiting For Autologous Stem Cell Transplantation

I. Torrea1, T. Castellanos1, M. Torrijos Zarcero1, Á. Palao Tarrero1, C. Rocamora1, B. Rodríguez Vega1, I. Louzao Rojas1. 1 Hospital Universitario La Paz, Psychiatry, Clinical Psychology and Mental Health, Madrid, Spain.

\title{
Backgrounds and Aims:
}

Hematologic diseases have a deep impact on physical, emotional and social aspects of patient's quality of life (1).

Prevalence of a psychiatric disorder among cancer patients ranges between $25-30 \%$. Prevalence is higher in patients who had been through a Stem Cell Transplantation (41-44\%) (2).

It is well known the influence of psychological aspects and stress on immune function, and the relationship between immune function and oncology.

To apply a multidisciplinary approach, which includes a Psychologist and/or Psychiatrist in the Hematology Units, is very important; and this idea is supported by recent studies in the field of Psychoneuroimmunology. This recent research has found that psychosocial variables influence the course and the recovery of the haemato-oncology disease (3).

The aim of this research is to study in a descriptive way some psychosocial variables related to Stem Cell Transplantation process in Hematologic cancer patients.

\section{Materials and Methods:}

We plan to collect data of every patient waiting for an Autologous Stem Cell Transplantation during 2019 and 2020 at Hospital Universitario La Paz, in Madrid, Spain.

We will collect descriptive information about Emotional Distress (Hospital Anxiety and Depression Scale, HADS), Quality of life (Functional Assessment of Cancer Therapy- Bone Marrow Transplantation, FACT-BMT), Mindfulness (Five Facets of Mindfulness Questionnaire, FFMQ), Self-compassion and Self-care ability (Self-Compassion ScaleShort Form, SCS-SF), Coping Strategies (COPE-Brief Form) and Optimism (unique question item). We will do so during the week prior transplantation.

\section{Results and Conclusions:}

We will analyze quantitative data in terms of means and standard deviations and qualitative data in terms of percentages and frequencies.

We will conduct Pearson correlations between outcome variables (Emotional Distress and Quality of Life) and process variables (Self-Compassion, Mindfulness, Coping strategies and Optimism).

We hypothesize that this group of population will have low levels of quality of life, and medium-high levels of emotional distress, and that these outcomes will correlate with way of coping, optimism, mindfulness and self-compassion. Indeed, we hypothesize that Mindfulness, Self-Compassion, Active Coping and Optimism will positively correlate with quality of life and negatively correlate with emotional distress.

It is necessary to care about emotional aspects of hematologic cancer patients in order to improve their global quality of life, and to help them in the course of the disease and the process of recovery.

\section{References:}

(1) Pulgar-Buendía, A. (2010). Aplicación del modelo biopsicosocial para la comprensión de la calidad de vida y el pronóstico en el cancer hematologico. [Aplication of the Biopsychosocial model to the understanding of the quality of life and prognosis in haemotological cancer]. Doctoral Dissertation. Spain: Universidad de Jaén.

(2) Sasaki, T., Akaho, R., Sakamaki, H., Akiyama, H., Yoshino, M., Hagiya, K., Atsumi, M. (2000). Mental disturbances during isolation in bone marrow transplant patients with leukemia. Bone Marrow Transplant, 25 (3): 315-8.

(3) McGregor, B. A., Syrjala, K. L., Dolan, E. D., Langer, S. L. \& Redman, M. (2013). The effect of pre-transplant distress on immune reconstitution among adult autologous hematopoietic cell transplantation patients. Brain Behav Immun 30, S142-8. 